\title{
An Axiomatization of the Nash Bargaining Solution
}

\author{
Geoffroy de Clippel $^{1}$
}

November 9, 2004

\begin{abstract}
I prove that the Nash bargaining solution is the only solution to satisfy 'Disagreement Point Convexity' and 'Midpoint Domination'. I explain how this improves previous results obtained by Chun (1990) and by Dagan et al. (2002).
\end{abstract}

\section{Introduction}

Dagan et al. (2002) prove that the Nash bargaining solution is the only solution to satisfy the following seven properties when there are exactly two players: 'Symmetry', 'Weak Pareto Optimality', 'Scale Covariance', 'Single-Valuedness in Symmetric Problems', 'Independence of Non-Individually Rational Alternatives', 'Twisting' and 'Disagreement Point Convexity'. I improve the result by showing that 1) The six first properties implies 'Midpoint Domination' (see Thomson, 1994, section 4.1), the converse being not true; 2) The Nash bargaining solution is the only solution to satisfy 'Disagreement Point Convexity' and 'Midpoint Domination'; 3) The last result remains valid with more than two players, provided that some regularity condition is imposed on the set of feasible agreements. My axiomatic result is closely related to Chun (1990). The proof being different, I am able to show among other things that his continuity axiom is redundant.

\section{Definitions}

Let $n$ be a positive integer and let $N:=\{1, \ldots, n\}$ be the set of players. A bargaining problem is a couple $(V, d)$ where $V \subseteq \mathbb{R}^{N}$ is the set of utility vectors that the players can achieve through cooperation and $d \in V$ is the utility vector that prevails in case of disagreement. The players have a strict interest to cooperate $(d<<v$ for some $v \in V)$ and the set $V$ of feasible agreements is compact,

\footnotetext{
${ }^{1}$ Chargé de Recherches FNRS. Department of Economics, Box B, Brown University, Providence RI 02912, USA. I thank Professors Hans Peters, Roberto Serrano and William Thomson for their very useful comments. The usual disclaimer applies.
} 
convex and non-level. Non-level means that the utilities are transferable above the disagreement point (of course not necessarily at a one to one exchange rate). If a vector is orthogonal to $V$ at some individually rational utility vector, then it is strictly positive. I also assume that $V$ is smooth when there are more than two players. This means that $V$ admits a unique supporting hyperplane at each utility vector that is Pareto optimal and individually rational.

A solution is a function that associates a nonempty set of feasible utility vectors to each bargaining problem. For instance, the Nash bargaining solution selects the utility vector that maximizes the product of the utility gains of the players over the set of feasible agreements that are individually rational, i.e.

$$
\Sigma_{N a s h}(V, d):=\arg \max _{\{v \in V \mid v \geq d\}} \prod_{i \in N}\left(v_{i}-d_{i}\right)
$$

\section{Main Result}

Here are two restrictions that could be imposed on a solution $\Sigma$. They are supposed to hold for each bargaining problem $(V, d)$.

Disagreement Point Convexity (DPC) If $\sigma \in \Sigma(V, d)$, then $\sigma \in \Sigma(V, \pi d+$ $(1-\pi) \sigma)$ for each $\pi \in] 0,1[$.

If $\sigma$ is a reasonable agreement for a bargaining problem $(V, d)$, then so should it remain for the modified problem $(V, \pi d+(1-\pi) \sigma)$. The solution has to be invariant to movements of the disagreement point towards the final agreement. The axiom was first introduced by Peters and van Damme (1991). Chun and Thomson (1990) apply a similar axiom imposing some linearity on the solution with respect to the disagreement point. I refer to these papers for justifications in terms of invariance with respect to resolution of the uncertainty about the disagreement point. Dagan et al. (2002) relate the axiom to properties of the non-cooperative bargaining models.

Midpoint Domination (MD) If $\sigma \in \Sigma(V, d)$, then $\sigma \geq \sum_{i \in N} a^{i}(V, d) / n$, where $a^{i}(V, d):=\arg \max _{\left\{v \in V \mid v_{-i} \geq d_{-i}\right\}} v_{i}$, for each $i \in N$.

If player $i$ had all the bargaining power, the outcome would be $a^{i}(V, d)$. A natural reference point when the players have equal bargaining abilities is obtained by mixing these extreme points, using a uniform probability distribution. It is only because of its lack of efficiency that the random dictatorship principle has to be amended. The objective of most bargaining solutions is to specify a way to split the remaining surplus when the midpoint is not Pareto optimal. For instance, the discrete Raiffa bargaining solution (see Luce and Raiffa, 1957, pages 136-137) considers the midpoint as a partial agreement and applies recursively the random dictatorship argument up to the Pareto frontier. The solution of Kalai and Smorodinski (1975) follows the direction defined by the disagreement point and the midpoint up to the Pareto frontier. I prove hereafter that the 
Nash solution itself satisfies the axiom. Though natural and powerful, MD has not been used frequently in the literature. The only references that I am aware of are Moulin (1983) and Chun (1990). I discuss these papers in the next section.

Theorem The Nash bargaining solution is the only solution to satisfy 'Disagreement Point Convexity' (DPC) and 'Midpoint Domination' (MD).

The proof is based on the following characterization of the Nash bargaining solution suggested by Harsanyi (1959). It is obtained by separating $V$ from the set of utility pairs whose Nash product is greater than its evaluation at the solution. Both sets are indeed convex.

Lemma (Harsanyi, 1959) Let $\sigma \in V$. Then, $\sigma$ is the Nash bargaining solution if and only if $\sigma>>d$ and there exists $\lambda \in \mathbb{R}_{++}^{N}$ such that

a. $\lambda . \sigma=\max _{v \in V} \lambda . v$

b. $(\forall(i, j) \in N \times N): \lambda_{i}\left(\sigma_{i}-d_{i}\right)=\lambda_{j}\left(\sigma_{j}-d_{j}\right)$.

The lemma can be interpreted as follows: the Nash bargaining solution is the only feasible agreement that satisfies simultaneously the utilitarian and the egalitarian objectives for some re-scaling of the individual utilities (see Shapley, 1969; Myerson, 1991, section 8.3).

Proof of the theorem: The Nash bargaining solution satisfies both DPC and $\mathrm{MD}$ as a consequence of the lemma. Indeed, the two conditions stated there implies that $\sigma=\sum_{i \in N} a^{i}\left(V^{\lambda}, d\right) / n$ where $V \subseteq V^{\lambda}:=\left\{x \in \mathbb{R}^{N} \mid \lambda . x \leq \lambda . \sigma\right\}$. On the other hand, $\lambda_{i}\left(\sigma_{i}-\left(\pi d_{i}+(1-\pi) \sigma_{i}\right)\right)=\pi \lambda_{i}\left(\sigma_{i}-d_{i}\right)$ for each $i \in N$.

Let $\Sigma$ be a solution that satisfies both DPC and MD, let $(V, d)$ be a bargaining problem and let $\sigma \in \Sigma(V, d)$. As $d$ is not weakly Pareto optimal, MD implies that $\sigma>>d$. Observe also that $\sigma$ is Pareto optimal. Otherwise, the condition $\sigma \in \Sigma(V, \pi d+(1-\pi) \sigma)$ imposed by $\mathrm{DPC}$ is incompatible with MD when $\pi$ is small. Let $\lambda \in \mathbb{R}_{++}^{N}$ be a vector that is orthogonal to $V$ at $\sigma$. Condition $a$ of the lemma is trivially satisfied. Combining DPC and MD, I have

$$
\sigma \geq \sum_{j \in N} \frac{a^{j}(V, \pi d+(1-\pi) \sigma)}{n}
$$

for each $\pi \in[0,1]$, which amounts to

$$
(n-1)\left(\sigma_{i}-d_{i}\right) \geq \frac{a_{i}^{i}(V, \pi d+(1-\pi) \sigma)-\sigma_{i}}{\pi}
$$

for each $i \in N$ and each $\pi \in[0,1]$. Taking the limit as $\pi$ tends to zero and using the fact that $V$ is smooth, I obtain

$$
(n-1)\left(\sigma_{i}-d_{i}\right) \geq \sum_{j \in N \backslash\{i\}} \frac{\lambda_{j}\left(\sigma_{j}-d_{j}\right)}{\lambda_{i}}
$$


for each $i \in N$. Hence,

$$
\lambda_{i}\left(\sigma_{i}-d_{i}\right) \geq \frac{\lambda \cdot(\sigma-d)}{n}
$$

for each $i \in N$. Taking the sum over $i$, I conclude that the inequality cannot be strict. The right term does not depend on $i$. Condition $b$ of the lemma is therefore satisfied.

I now prove the theorem for two-player bargaining problems without assuming that $V$ is smooth. Let $v: \mathbb{R} \rightarrow \mathbb{R}$ be the concave function that represents the Pareto frontier of $V$, i.e. $x_{2}=v\left(x_{1}\right)$ if and only if $x$ is strongly Pareto optimal in $V$. Starting from equation (3) and taking the limit as $\pi$ tends to zero, I obtain

$$
v_{r}^{\prime}\left(\sigma_{1}\right) \leq \frac{d_{2}-\sigma_{2}}{\sigma_{1}-d_{1}} \leq v_{l}^{\prime}\left(\sigma_{1}\right)
$$

where $v_{l}^{\prime}$ and $v_{r}^{\prime}$ denote respectively the left and right derivatives of $v$. Hence condition $a$ of the lemma is satisfied with $\lambda=\left(\sigma_{2}-d_{2}, \sigma_{1}-d_{1}\right)$. Condition $b$ is trivially satisfied as well.

The Nash bargaining solution does not necessarily satisfy MD when $V$ is not required to be non-level. For instance, let $n=2$, let $d=(0,0)$ and let $V$ be the convex hull of the vectors $(0,0),(1,1)$ and $(2,0)$. The Nash bargaining solution is $\{(1,1)\}$, but $\left[a^{1}(V, d)+a^{2}(V, d)\right] / 2=(3 / 2,1 / 2)$.

The Nash bargaining solution is not the only solution to satisfy DPC and MD when $V$ is not smooth and there are more than two players. For instance, let $n=3$, let $\hat{V}$ be the convex hull of the vectors $(0,0,0),(15,0,0),(0,15,0)$, $(10,10,0)$ and $(0,0,10)$, and let $\Sigma$ be the solution defined as follows: $\Sigma(V, d):=$ $\Sigma_{\text {Nash }}(V, d) \cup\{(5,5,5)\}$ if $(V, d)=(\hat{V}, \pi(0,0,0)+(1-\pi)(5,5,5))$ for some $\pi \in[0,1]$ and $\Sigma(V, d):=\Sigma_{N a s h}(V, d)$ for each other bargaining problem $(V, d)$. Notice that $(5,5,5) \notin \Sigma_{N a s h}(V, \pi(0,0,0)+(1-\pi)(5,5,5))$ for each $\left.\pi \in\right] 0,1[$. The solution $\Sigma$ obviously satisfies DPC. It also satisfies MD because $(5,5,5) \geq$ $\sum_{i=1}^{3} a^{i}(\hat{V}, \pi(0,0,0)+(1-\pi)(5,5,5)) / 3$ for each $\pi \in[0,1]$. Indeed, $a^{1}=(5+$ $10 \pi, 5(1-\pi), 5(1-\pi)), a^{2}=(5(1-\pi), 5+10 \pi, 5(1-\pi))$ and $a^{3}=(5(1-$ $\pi), 5(1-\pi), 10-5(1-\pi))$. The theorem may be adapted in order to axiomatize the Nash bargaining solution for non-smooth problems with more than two players by focusing on single-valued solutions that are continuous. It should be noted though that the smoothness assumption is a mild and natural regularity condition.

\section{Related Literature}

I start by citing some related results that are not directly comparable. Moulin (1983) (see Thomson, 1994, section 4.1) shows that the Nash bargaining solution is the only single-valued solution to satisfy MD and 'Independence over Irrelevant Alternatives'. I proved that a similar result holds with DPC instead of IIA. Peters and van Damme (1991) apply a fixed-point argument in order to show that the Nash bargaining solution is the only single-valued solution 
to satisfy DPC, 'Disagreement Point Continuity', 'Scale Covariance', 'Symmetry', 'Efficiency' and 'Independence of Non-Individually Rational Alternatives'. I obtained a similar result with MD replacing the five last axioms, allowing for multi-valued solutions and restricting my attention to smooth problems when there are more than two players.

Chun (1990, theorems 1 and 3) uses MD together with axioms that are slightly stronger than DPC ('Weak Disagreement Point Linearity' and 'Linearity', see also Chun and Thomson, 1990) in order to characterize the Nash bargaining solution. Nevertheless, he imposes in addition axioms of continuity and optimality. He also restricts his attention to single-valued solutions. I show that these properties are redundant, at least for smooth problems when there are more than two players. Chun's proof depends heavily on the continuity axiom (even in case of smooth problems) as he first proves his results for polyhedron problems and then applies a limit argument. The continuity axiom is especially annoying for an approach whose objective is to work with fixed feasible sets, imposing restrictions involving variations of the disagreement point only.

I devote the rest of the section to explain why my theorem improves the previous result obtained by Dagan et al. (2002). Their argument is valid only for bargaining problems with two players. They prove under this assumption that the Nash bargaining solution is the only solution to satisfy DPC along with the following six axioms that are supposed to hold for each bargaining problem $(V, d)$.

Symmetry A bargaining problem $(V, d)$ is symmetric if $d_{1}=d_{2}$ and $V=$ $\left\{\left(v_{2}, v_{1}\right) \mid v \in V\right\}$. If $(V, d)$ is symmetric, then $\Sigma(V, d)=\left\{\left(\sigma_{2}, \sigma_{1}\right) \mid \sigma \in \Sigma(V, d)\right\}$.

Weak Pareto Optimality Any element of $\Sigma(V, d)$ is weakly Pareto optimal.

Scale Covariance $\Sigma(\alpha V+\beta)=\alpha \Sigma(V, d)+\beta$, for each $\alpha \in \mathbb{R}_{++}^{2}$ and each $\beta \in \mathbb{R}^{2}$

Single-Valuedness in Symmetric Problems If $(V, d)$ is symmetric, then $\Sigma(V, d)$ is a singleton.

Independence of Non-Individually Rational Alternatives If a bargaining problem $\left(V^{\prime}, d\right)$ is such that $\left\{v \in V^{\prime} \mid v \geq d\right\}=\{v \in V \mid v \geq d\}$, then $\Sigma\left(V^{\prime}, d\right)=\Sigma(V, d)$.

Twisting Let $\sigma \in \Sigma(V, d)$, let $i \in\{1,2\}$ and let $\left(V^{\prime}, d\right)$ be a bargaining problem such that $V \backslash V^{\prime} \subseteq\left\{x \in \mathbb{R}^{2} \mid x_{i}>\sigma_{i}\right\}$ and $V^{\prime} \backslash V \subseteq\left\{x \in \mathbb{R}^{2} \mid x_{i}<\sigma_{i}\right\}$. Then, $\sigma_{i}^{\prime} \leq \sigma_{i}$ for some $\sigma^{\prime} \in \Sigma\left(V^{\prime}, d\right)$.

These axioms together imply $\mathrm{MD}$ as the following argument shows. Let $(V, d)$ be a bargaining problem and let $\sigma \in \Sigma(V, d)$. Suppose that $\sigma \neq u^{1}(V, d)$. Let $V^{\prime}$ be the set of individually rational utility pairs that is bounded above by the line going through the points $\sigma$ and $u^{1}(V, d)$. By the first four ax- 
ioms, $\Sigma\left(V^{\prime}, d\right)=\left\{\left(\frac{d_{1}+u_{1}^{1}(V, d)}{2}, x\right)\right\}$ for some $x \in \mathbb{R}$. By 'Independence of NonIndividually Rational Alternatives', $\sigma \in \Sigma(\hat{V}, d)$ where $\hat{V}$ is the set of individually rational utility pairs that belong to $V$. By 'Twisting', $\sigma_{1} \geq\left(d_{1}+u_{1}^{1}(V, d)\right) / 2$. A similar argument implies that $\sigma_{2} \geq\left(d_{2}+u_{2}^{2}(V, d)\right) / 2$ if $\sigma \neq u^{2}(V, d)$. It is then easy to conclude.

On the contrary, MD does not imply the above axioms. In fact, it does not even imply any of them. Here are examples of solutions that satisfy MD but violates some of the above axioms. The solution that is obtained by projecting vertically (upward) the midpoint on the Pareto frontier of the feasible set does not satisfy 'Symmetry', the solution that selects the midpoint does not satisfy 'Weak Pareto Optimality', the solution that equally split the surplus above the midpoint does not satisfy 'Scale Covariance', the solution that selects the set of Pareto optimal utility vectors that dominates the midpoint does not satisfy 'Single-Valuedness in Symmetric Problems', the solution that maximizes the product of the utility gains with respect to the downward vertical projection of the disagreement point on the boundary of the feasible set and over the set of feasible utility vectors that Pareto dominate the midpoint does not satisfy 'Independence of Non-Individually Rational Alternatives', and finally the solution that selects the utility vectors on the Pareto frontier that are the closest to the midpoint does not satisfy 'Twisting'.

Notice finally that MD is defined for a given bargaining problem although 'Scale Covariance', 'Independence of Non-Individually Rational Alternatives' and 'Twisting' involve comparisons between different bargaining problems with different feasible sets.

\section{References}

[1] Chun, Y., 1990, Minimal Cooperation in Bargaining, Economics Letters, $34,311-316$.

[2] Chun, Y. and W. Thomson, 1990, Nash Solution and Uncertain Disagreement Points, Games and Economic Behavior, 2, 213-223.

[3] Dagan, N., O. Volij and E. Winter, 2002, A Characterization of the Nash Bargaining Solution, Social Choice and Welfare, 19, 811-823.

[4] Harsanyi, J. C. 1959, A Bargaining Model for the Cooperative n-Person Games, In: A. W. Tucker and R. D. Luce (Eds), Contributions to the Theory of Games IV, Princeton University Press, 325-355.

[5] Kalai, E. and M. Smorodinsky, 1975, Other Solutions to Nash's Bargaining Problem, Econometrica, 43, 513-518.

[6] Luce, R. D. and H. Raiffa, 1957, Games and Decisions, New York: Wiley.

[7] Moulin, H., 1983, Le choix social utilitariste, Ecole Politechnique DP. 
[8] Myerson, R. B., 1991, Game Theory (Analysis of Conflict), Harvard University press.

[9] Nash, J. F., 1950, The Bargaining Problem, Econometrica, 18, 155-162.

[10] Peters, H. and E. van Damme, 1991, Characterizing the Nash and Raiffa Bargaining Solutions by Disagreement Point Axioms, Mathematics of Operation Research, 16, 447-461.

[11] Shapley, L. S., 1969, Utility Comparison and the Theory of Games, In: La Decision, Editions du CNRS, 251-263.

[12] Thomson, W., 1994, Cooperative Models of Bargaining, In: R. J. Aumann and S. Hart (eds) Handbook of Game Theory, North Holland, 1237-1284. 